\title{
Ways to improve the efficiency of multifunction electroprospecting instruments for mining exploration
}

\author{
I. Ingerov \\ Advanced Geophysical Operations and Services Inc. \\ (AGCOS)
}

\begin{abstract}
The idea of developing a multifunction EM instruments implementing many ground EM methods with a common ideology of processing and interpreting the data has been held by geophysicists from the 1980 s of the last century. At the turn of the 1980s and 1990s several companies (Phoenix, Zonge) managed to achieve positive results. However, the equipment of the time remained bulky, heavy, was expensive and low productive. The modern development of electronics and computer technology allows to fully realize the idea of versatility and combine in a single instrument seemingly not possible to be joined in the past MT and VES functions. Portable, multifunction equipment is offered based on 4- and 8-channel EM receivers, with flexible configuration of magnetic and electrical channels. Equipment can effectively implement virtually all ground electroprospecting methods (excluding ground penetrating radar). A set of equipment includes all the necessary elements of hardware and software for productive field work including field recording, processing of field data and interpretation (including complex). That is, system may include one or more wideband EM receivers, wideband current source (transmitter), wideband sensors of electric and magnetic field components, precision tripods for mounting magnetic sensors, set of accessories, and set of software for the operation of equipment, processing, and interpretation of the field data.
\end{abstract} Keywords-multifunction; electroprospecting instruments; mining
exploration

\section{INTRODUCTION}

For mining and groundwater exploration, it is often necessary to combine several electroprospecting methods for a complete solution of the problem. Typically, hardware and software for different geophysical methods is manufactured by different companies with different ideology. In addition to the inconvenience associated with the use of hardware and software with different ideology, there is also a serious drawback associated with the price indices for both the customer and the contractor. The idea of developing a multifunction apparatus implementing many ground EM methods with a common ideology of processing and interpreting the data has been held by geophysicists from the 1980 s of the last century. At the turn of the 1980s and 1990s several companies managed to achieve positive results. The company has created a Phoenix 4th generation multifunctional devices based on V-5. The device could implement resistivity, TDEM (1 channel), IP(8 channels), CSAMT, MT and AMT. Implementation of the latter two methods required additional external amplifiers. Working with resistivity methods using this instrument was rather expensive. In general, the effective implementation in a single set of instrumentation of the MT, AMT, CSAMT, IP (all versions) methods made the $\mathrm{V}-5$ unit best receiver of its time. Zonge's GDP-32 receiver enjoys well-deserved prestige among geophysicists with high performance in the implementation of methods CSAMT and IP. However, it was not intended to implement the MT and AMT methods, which of course reduced its versatility. The designers of the proposed innovative multifunction EM instruments took into account the positive features of these devices, as well as their more recent clones to create a modern set of super multifunction electroprospecting equipment. The engineers set themselves the task of effective implementation in one set of equipment of all known ground electroprospecting methods (except GPR). For the base to create the instrument were adopted technical requirements for devices implementing methods MT and AMT. That is:

- High sensitivity.

- Low noise level.

- A wide frequency range.

- Wide dynamic range.

An important new requirement was flexible configuration of magnetic and electrical channels. In parallel with portable recorders were created:

- Wideband geophysical current source.

- Low-noise non-polarizing electrodes.

- Highly sensitive wideband induction coil sensors of magnetic field components.

- Precision tripods for accurate installation and alignment of magnetic sensors on any soil or terrain.

- $\quad$ Set of accessories for the field work.

- Software for the operation of equipment, processing, and interpretation of the field data. 

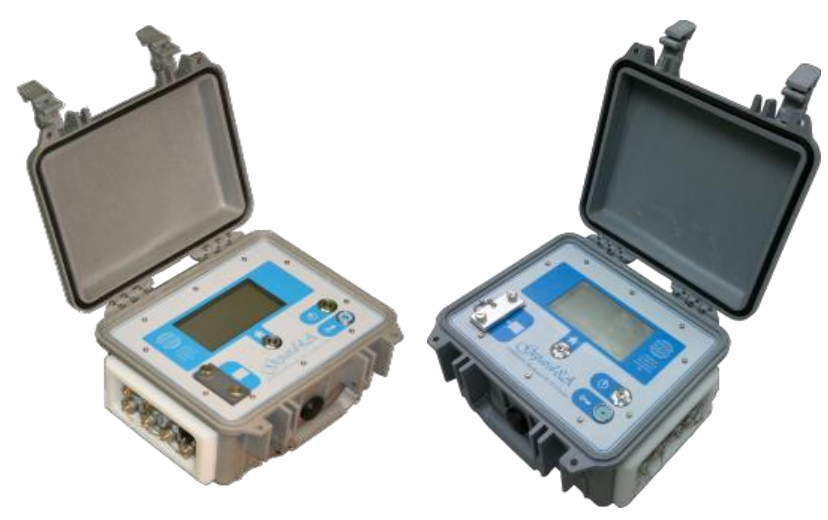

Fig. 1. Multifunction geophysical EM receivers; A) 4-channel GEPARD-4A; B) 8-channel GEPARD-8A.

\section{Multifunction EM Receivers}

Receivers are built using a 4- or 8-channel base (Fig.1). To provide a convenient interface, the latest devices are equipped with a sensitive screen that allows for quick and easy selection of prospecting methods and parameters of EM field recording. The signal and all essential operational information is entered onto a removable SD card, which ensures a quick transfer of data to a computer for processing and interpretation. The magnetic and electric channels are interchangeable on both devices, at a command from the screen. This provides flexibility in selecting field installations for recording EM fields. Electroprospecting methods deployed by device are grouped according to similar properties. The first group includes methods using the natural EM field of the earth: SP, MT, AMT, MVP. The next group of methods uses frequency domain controlled source and includes the following methods: CSAMT, FDEMS, SIP, phase, and amplitude IP. The third group of methods includes methods that use time domain signals: TDIP, FastTEM, MulTEM, LowTEM. The fourth group of methods includes methods of resistance: EP, VES, Electrotomography.

Devices are equipped with a GPS receiver, which allows for the automatic registration of survey site coordinates, as well as time stamps when recording time series. Devices also have a flexible selection of data sampling rates, which gives field researchers a convenient instrument for identifying useful signals and suppressing EM field noise.

\section{Wideband Transmitter of Electrical CuRRENT}

The portable generator AT-100 (Fig.2) has a 100W output and a range of functions of constant current in the interval of $0.001-1.0 \mathrm{~A}$. The current function is selected automatically based on load resistance. The generator has a dense grid of generated frequencies in the range of $0.001-50,000 \mathrm{~Hz}$. The frequency grid consists of 5 rows, derivable from: $1 \mathrm{~Hz}$, $1.22 \mathrm{~Hz}, 1.33 \mathrm{~Hz}, 1.5 \mathrm{~Hz}, 1.66 \mathrm{~Hz}$. The frequency is changed by multiplying the respective row by $2 n$, where $n$ can have a negative or a positive integer value. The frequency can be changed manually using the screen of the transmitter or the order of the generated frequencies and the generation time of each of them can be stored on a removable SD card. In this case, the transmitter will operate in an automatic mode.

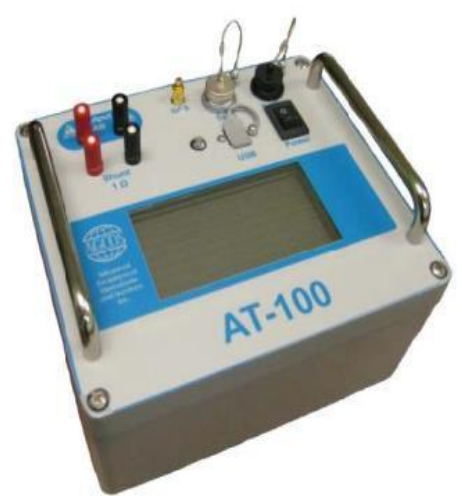

Fig. 2. Multifunction current source for geophysical EM techniques AT-100.
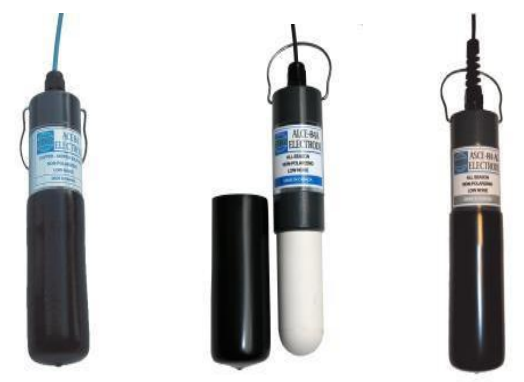

Fig. 3. Low-noise non-polarizing electrodes; A) ACE-84 (copper); B) ALCE-84A (lead); C) ASCE-84AG (silver).

\section{LOW-NOISE NON-POLARIZING ELECTRODES}

To measure the electric component of the EM field, grounded electric lines of $5-100 \mathrm{~m}$ are used. Lines are grounded using low-noise non-polarizing electrodes. These electrodes have hermetic housing with a custom-formed porous membrane and a contact wire, to which is soldered a metal rod encased in a special gel containing the salt of the given metal. Electrodes (Fig.3) are manufactured on the basis of copper, lead, and silver. The silver electrodes are the most stable and environmentally friendly but they are distinguished by a relatively high cost and the complexity of manufacturing a stable gel fill. The large effective area of the special form membrane allows for low resistance grounding, which promotes high-quality measurements.

\section{INDUCTION COILS AND AIRCOILS (LOOPS) For MEASURING MAGNETIC FiELd COMPONENTS}

For methods using EM field frequency domain, induction coils have been used over the past two decades as EM field sensors. Each coil has a magnetic core, a multi-turn coil, a coil gauge, an electric shield and a low-noise wideband preamplifier board. Coils are connected to the device using noise-immune cables. Two sensors of different construction (Fig.4) cover the entire frequency range of the exploration. AMS-15 has a frequency range of $50,000-1 \mathrm{~Hz}, \mathrm{AMS}-37$ has a frequency range of $500-0.0001 \mathrm{~Hz}$. The sensors are hermetically sealed and can be buried underground, however a more effective installation is via specialized precision tripods (see below). 


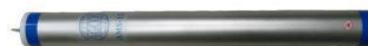

Fig. 4. Induction coil magnetic sensors; A) AMS-15, B) AMS-37.

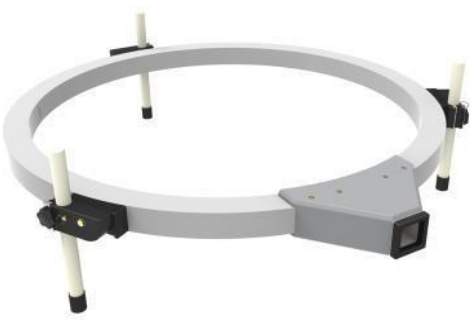

Fig. 5. MTEM-200 airloop sensor for TDEM work.

To measure the derivative of the vertical component of the magnetic induction by time, airloop sensors equipped with a preamplifier are used. The diameter of the frame for FastTEM is $60 \mathrm{~cm}$ (50 turns). The diameter of the frame for MulTEM is $1 \mathrm{~m}$ (100 turns), as shown in Fig.5, and the side of the square loop for Low TEM is $25 \mathrm{~m}$ (50 turns) ${ }^{[1]}$.

\section{PRECISION FIELD TRIPODS}

Precision field tripods are a unique instrument that appeared at the beginning of this century. This tool enabled a significant expansion in the scope of application of methods AMT, MVP, MT, CSAMT, as well as, in some cases, to significantly increase the productivity of field teams ${ }^{[3,4,5]}$. 3component tripods are the most effective, which can quickly and easily transform from portable to working configuration and vice versa (1-3 minutes), as well as be easily installed on any terrain. Single component tripods (Fig.6) can be used as effectively, however productivity increases derived from these are not as significant.

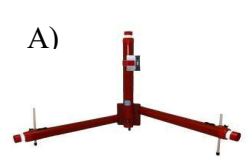

C)

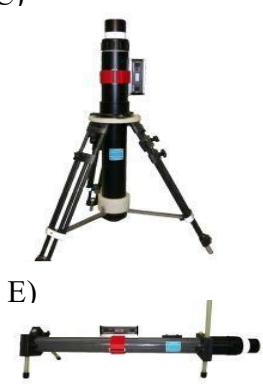

AMT Tripods
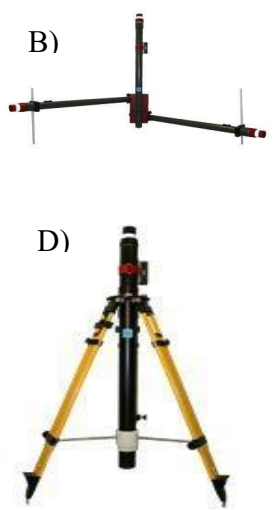

F)

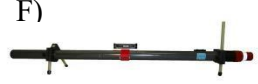

MT Tripods
Fig. 6. Precision field tripods for induction coil magnetic sensors; A) TRI3/30; B) TRI-3/50; C) TRI-1/30; D) TRI- 1/50; E) TRI-1/30/1; F) TRI-1/50/1.

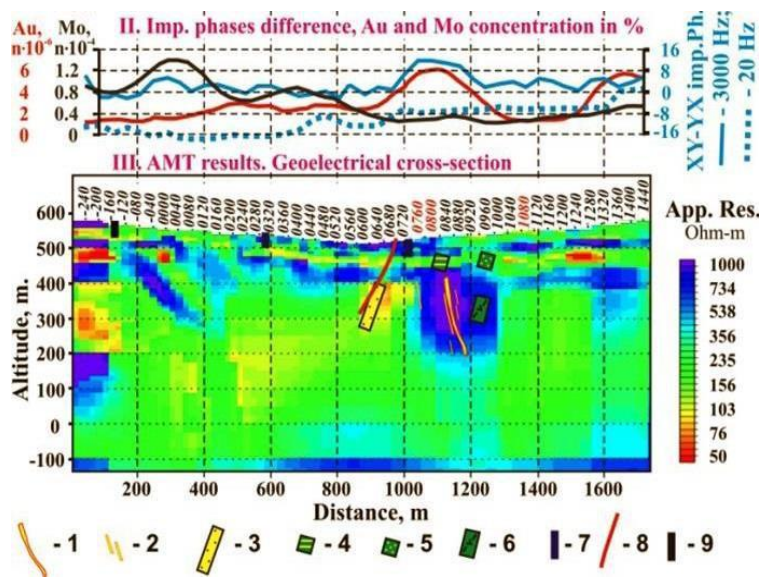

Fig. 7. Survey result comparison. II - ground geochemistry results and difference between XY and YX impedance phase for 3000 and $20 \mathrm{~Hz}$; III Geoelectrical cross-section (2D inversion of AMT-MVP data); Legend for III: general drill holes results (1-6): 1 - main gold ore vein, 2 - thin ore veins, 3 felsic ach tuff, 4 - strongly altered basalt lava, 5 - strongly alternated basalt tuff, 6 - andesite - basalt lava; geological mapping information (7-9): 7 - dyke position, 8 - fault zone position, 9 -andesite - rhyolite contact.

\section{FIELD APPLICATION EXAMPLES}

One of the successful examples of precision field tripods application is recent gold exploration survey in Chukotka region using AMT method ${ }^{[2]}$. Results of $2 \mathrm{D}$ inversion were done for all profiles using WinGLink software. Geoelectrical cross-section for profile 2 has been shown at Fig.7. $\rho, \varphi$ of TE-mode and TM-mode in frequency range from 10,000 to $50 \mathrm{~Hz}$ and tipper amplitude (Ta) in frequency range from 10,000 to $3,000 \mathrm{~Hz}$ was used for joint $2 \mathrm{D}$ inversion. MRS was achieved at $1.3 \%$. Above Geoelectrical section the graphs of geological and geophysical parameters are shown. On the section at $200 \mathrm{~ms}$ west from the fault allocated high resistivity zone in the depth range $100-600 \mathrm{~m}$. In the center of this area is drilled gold-bearing quartz vein. Over a high resistivity zone is also observed: positive gold, silver and arsenic anomaly in subsoil, positive anomaly of the phase difference of the longitudinal ( $\varphi x y)$ and transverse ( $\varphi$ yx) components, as well as local anomaly constant magnetic field of the Earth (T). Such features can be regarded as the search criteria for the blind auriferous quartz veins. Most pronounced over the auriferous vein zone manifested negative anomaly phase of the tipper (blue curve on Fig.7). Noteworthy negative tipper phase values in the eastern part of the tipper profile (station 1280-1400). According to the results of 2D inversion there, at depths of $100-300 \mathrm{~m}$ high resistivity is detected as well as the gold anomaly in subsoil. All of this can be attributed to this anomalous object on promising gold ore mineralization. In the western part of the profile (around site-100) also observed negative anomaly tipper phase and Magnetic field of the Earth. There are also appearances of vertical inhomogeneity on high the high resistive Geoelectrical section as well and as negative anomaly tipper phase and constant magnetic field of the Earth. 
The positive anomaly of molybdenum (little bit shifted to the east is present), which is typical for under ore body cut of gold-silver epithermal columns.
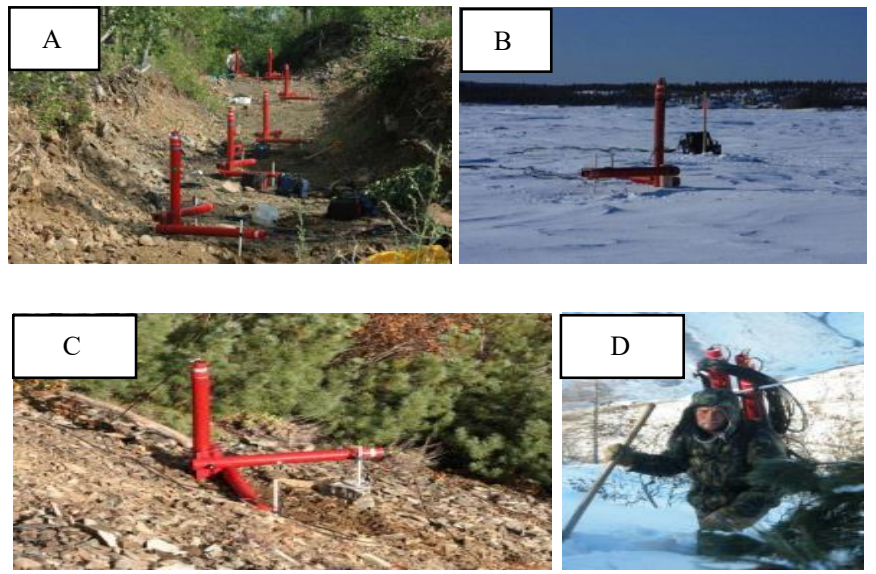

Fig.8. Application of precision tripods for induction magnetic sensor installation during field surveys: A) gold exploration near Baikal lake, Russia; B) exploration for sulphide ore, Northern Canada; C) investigations at Patom Crater, Russia; D) equipment set for MVP method in transportation mode.

\section{VIII.CONCLUSIONS}

- A compact, portable multi-function electromagnetic system is designed and produced.
- The system includes all the required elements for conducting field work, processing field data and its interpretation.

- The system is distinguished by 4- and 8-channel receivers with a flexible configuration of magnetic and electric channels.

- Implementation of three-axis precision tripods for magnetic sensor installation allows productive field work to be carried out on any terrain.

- The system permits the use of the overwhelming majority of known electroprospecting methods.

\section{REFERENCES}

[1] Ingerov, O., 2005, Application of electroprospecting for hydrocarbon exploration, Notes of the (St. Petersburg) Mining Institute, 162, 15-25.

[2] Ermolin, E., et. al. 2014, Gold exploration in Chukotka region by using audimagnetotellurics, Extended Abstract, 22nd EM Induction Workshop, Weimar, Germany, August 24-30, 2014.

[3] Ingerov, I., 2011, Method of multifrequency magnetovariational profiling (MVP), EMS-2011, St. Petersburg, Russia, Abstracts, Vol.2, 449-454.

[4] Ingerov, I., 2014, Multifunction electroprospecting instruments for complex investigations, mapping, monitoring and exploration in a wide depth interval, Extended Abstract, 22nd EM Induction Workshop, Weimar, Germany, August 24-30, 2014.

[5] Ingerov, O., 2011, Current trends in the development of electroprospecting hardware set for ground and marine surveys, EMS2011, St. Petersburg, Russia, Abstracts, Vol.1, 86-101. 